\title{
Screening of antagonistic yeast for biological control activity against anthracnose (Colletotrichum gloeosporioides) in 'Frangi' papaya.
}

\begin{abstract}
Postharvest decay causes decreased in both quantity and quality of fruits. It is also one of the major constraints in papaya production and exports. Anthracnose caused by Colletotrichum gloeosporioides was the most prevalent postharvest fungal disease attacking 'Frangi' papaya, where disease incidence and severity was recorded as 90-98 and 25-38\%, respectively and the magnitude increased as the fruits ripened. Conventional fungicide treatment is losing popularity due to high residual problem and detrimental to the environment. Thus, a study was conducted to look for a suitable strain of yeast that can substitute fungicides in controlling the disease with the objectives to select and test the ability of epiphytic yeast isolated from papaya fruit, leaf, petiole and trunk in controlling anthracnose onset after harvest. A total of 110 different strains of yeasts were obtained and 29 yeasts isolated showed antagonistic activities against C. gloeosporioides in vitro. Out of 29 yeasts, only five strains YK, YC, YT, YA and YW showed highest percentages inhibition of radial growth $(70.3,60.8$, $59.5,59.5$ and $59.5 \%$, respectively) in dual culture assay. Further assays were done in vitro and in vivo in order to confirm the antagonist activities of the yeasts by referring to percentage of disease reduction and inhibition of mycelial growth. Only two strains, YA and YK showed higher potential in controlling anthracnose in in vivo and in vitro test. Based on these results, additional studies on identification and modes of action of the most potential antagonistic yeast(s) to control postharvest anthracnose disease will be conducted.
\end{abstract}

Keyword: Biocontrol; Antagonist yeast; 'Frangi' papaya; Anthracnose. 